\title{
Students' Perception on Effectiveness of Teaching Learning Process at Higher Secondary Schools in Bahawalpur, Punjab
}

\author{
${ }^{a}$ Muhammad Zeeshan, ${ }^{b}$ Akhtar Ali, ${ }^{c}$ Najam Ul Kashif \\ ${ }^{a}$ PhDScholar, Department of Education, The Islamia University of Bahawalpur, Pakistan. \\ Email: zeeshanabbasi703@gmail.com \\ ${ }^{\mathrm{b}}$ Dean, Faculty of Education, The Islamia University of Bahawalpur, Pakistan. \\ Email: akhtariub@hotmail.co.uk \\ ${ }^{c}$ Assistant Professor, Department of Education, The Islamia University of Bahawalpur, Pakistan. \\ Email: najamulkashif@gmail.com
}

\begin{tabular}{l}
\hline ARTICLE DETAILS \\
\hline History: \\
Accepted 10 April 2021 \\
Available Online May 2021 \\
\hline Keywords: \\
Effectiveness, Intermediate, \\
Higher Secondary School \\
JEL Classification: \\
P36, I21 \\
\hline
\end{tabular}

DOI: $10.47067 /$ real.v4i2.143

\section{ABSTRACT}

The major objective of the article is to find out the students' perception on effectiveness of teaching learning process at higher secondary schools in Bahawalpur, Punjab. A randomly selected group of 545 students of intermediate classes (1st and 2nd year) from the six government higher secondary schools of Bahawalpur district responded on a four points agree/disagree Likert type scale through personal visits of the researcher to the data sights. Seven factors (Infrastructure, nature of relationship, teaching learning process, school environment, school management, discipline and co-curricular activities) solution with $47 \%$ of common variance was accepted as a result of Principle component analysis with Varimax rotation. All the factors explained $47 \%$ of total variance. Cronbach Alpha value remained more than 0.7 for all the cases. Strongly agreed and agreed responses on each item of the factor and then factor on the whole merged together to give rise agree frequency whereas addition of strongly disagree and disagree frequencies resulted disagree set of responses. The percentages of both the set of responses were calculated. Independent sample t test and one way ANOVA were run to work out the demographic effects. The results showed that more than sixty percent students were agreed about actively participating in learning activities, teachers help students to solve their learning problems, practice new skill of teaching in the classroom, institution building is neat and clean, priority of school is cleanliness, IT sources are sufficiently used in the school, coordinate with parents regarding learning of students and use variety of co-curricular activities for developing personality of students.

(C) 2021 The authors. Published by SPCRD Global Publishing. This is an open access article under the Creative Commons AttributionNonCommercial 4.0

Corresponding author's email address: najamulkashif@gmail.com 


\section{Introduction}

It is generally acknowledged that the socioeconomic development of nations commonly depends on effectiveness of its education system. Usually dedicated leadership, commitment of staff with academic excellence, their attitude towards teaching-learning process, physical facilities, learning environment and co-curricular activities are considered as the major indicators of educational effectiveness (Barton, 2005; Brophy, 2004; Fredericks, Blumenfeld \& Paris, 2004). Normally students attend the school to enhance their knowledge and skill. As a result they develop their personality, polish their leadership abilities, promote creativity, problem solving skill, and improve their confidence and analytical behavior. Generally consistency between goals and academic achievement, friendly environment with caring attitude, mutual respect and academic efforts are major ingredients for the promotion of such type of things (Saleem, 2010; Blair, 2002 \& Wallin, 2003).

Previous researchers (Reynolds, Teddlie, Creemers, Scheerens, 20oo; Rutter \& Maughan, 1979) indicate that an effective school has a supportive learning environment, student satisfaction with the teaching-learning process, their social well-being and relationship between educational goals and learning results. In this sense, a school's effectiveness is the degree to which a school's established goals and objectives are met in terms of the quantity, quality, equality and relevance of education (Yusuf and Alabi, 2013).

In Pakistan schooling is divided into primary, middle, secondary levels. Secondary education is further divided into two levels level grade 9 and 10 (secondary classes) and level 2 grade 11 and 12 (higher secondary classes) (Ali et al., 2016). Secondary school is a significant component of school education. It provides middle level work force for the economy as well as acts as a feeder for higher education. The Intermediate level of education provides entry to university. At this stage the students are at the peak of their emotional development. They usually set their future goals and develop strategies to meet the targets. Generally suitable discipline is required to reach the goals. Therefore, the significance of intermediate level education institutions cannot be overlooked.

According to Pakistan Education Statistics 2016-17, there are 5,130 inter colleges and higher secondary schools to facilitate higher secondary schools students in Pakistan. The share of these institutions in the total education system of the country is about $2 \%$. Of these, 39\% (1998) of the organizations are active in the public sector. The total number of students enrolled in higher secondary schools / inter college is 1,583 million, of which 1,396 million (88\%) are in the public sector. The total enrollment of boys in higher secondary schools / inter colleges schools is 0.970 million (61\%), while the enrollment of girls is 0.612 million (39\%). 120,336 teachers work in the country's higher secondary schools / inter colleges educational level. Over time, a very large number of secondary schools have been observed. In 2012-13, there were 1,404 secondary schools in the country and in 2016-17, after five years, we have 1698 higher secondary schools in our public sector education system (Pakistan Education Statistics 2016-17).

It appears from the above discussion that both higher secondary schools and intermediate colleges are responsible for intermediate education in Pakistan. Higher secondary schools enjoyed a rapid growth during the last couple of decades with the growing share in the system. Hence the need merged to investigate these institutions. This article is an attempt to analysis the effectiveness of higher secondary schools in district Bahawalpur of Punjab province. 


\section{Sampling}

According to school census 2018 of school education department, government of Punjab, Pakistan, 751 higher secondary schools were functioning in Punjab. Bahawalpur is among one of the 36 districts of Punjab Province. Being a resident of this area the researcher decided to conduct in depth analysis of the effectiveness of these institutions. As per Government of Punjab School Education Department website information 27 higher secondary schools were located under the administrative jurisdiction of this district in 2021. Out of these 15 institutions were female. Cluster sampling was exercised to draw 12 institutions from targeted population from the strata of male and females. Equal representation was given to gender in the choice of institutions. Total number of higher secondary enrolled students rests on 7903 (Govt. of Punjab, 2018). A random selection of 45 students of higher secondary classes was made from each of the sampled school through stratified sampling technique. Total sample size was 560 .

\section{Research Tool}

A four point agree-disagree Likert type rating scale was used as research tool for data collection. An extensive literature survey was made to identify the relevant measuring instruments. The related instruments were either downloaded from the websites or picked from the research articles or theses. In this way a pool of the relevant items was created. Moreover, some items were generated through extensive literature survey. A group of five experts of both language and the relevant field reviewed these items for establishing face as well as content validity of the research tool.

The item approved by $80 \%$ of the experts retained on the questionnaire. Three open ended items were included at the end of the data collection tool for exploring the views of the participants. A four points Likert Scale ranging from strongly agree to strongly disagree was attached to each item for recording the responses. An introductory paragraph explaining major objective of the study and procedure for how to respond the item was also added at the beginning of the research tool. This set of items was administered to a sample of 250 respondents. Three separate data sheets were generated in SPSS for recording the responses.

A coding scheme was prepared for assigning codes ranging from 1-4 for strongly disagree to strongly agree range of scale. After coding the data was entered independently by three persons in data sheets. A time to time matching of the data from each of the data sheet was made for resolution of discrepancy, data cleaning and validating purpose. The item having higher than the total tool Cronbach Alpha value calculated through if item deleted test was excluded from the tool.

A Principle Component Analysis with Varimax rotation was conducted on the data set generated as a result of the pilot testing. Seven factors solution was accepted with the explanation of $47 \%$ of the common variance of the responses. These seven factors include: infrastructure (item number 57, 58, $59,60,61,62,63$ ), nature of relationship (question number 53, 54, 55, 56,), teaching learning process (item number 1, 2, 3, 4, 5, 6, 7, 8, 9), school environment (item number 12, 14, 16, 17, 19, 20, 21), school management (item number 22, 23, 24, 25, 26, 27), discipline (item number 31, 32, 33, 34,35, 36, 37) and co-curricular activities (item number 64, 65, 66, 67, 68). Demographic information was added to the research tool. Cronbach Alpha value remained more than 0.7 for all the individual factors and .897 for the tool on the whole. The final questionnaire was consisted of 45 items. 


\section{Data collection and Analysis}

The researcher visited the data sights to administer the research tool to the respondents. The Principal of each school was requested to access the respondents for getting the research tool filled. After getting the permission a copy of the research tool was given to each respondent for entering the data. They were given enough time to record the responses in the data collection tool. Total 545 filled questionnaires received back to the researcher within the two months allocated time for data collection. The response rate was $97 \%$. Keeping in view the already described coding scheme the data was entered independently by the three persons in three separate data sheets of SPSS. Data cleaning and validating process was completed accordingly. Cronbach Alpha value for the whole data set was .876 .

A principal component analysis with Varimax rotation again conducted on the data set. The items having factor loading of above .3 and Eigen value greater than one retained on the factors. Seven factors solution was accepted. $47 \%$ common variance was explained by the factors. These factors include Infrastructure, management, student-teachers relationship, teaching strategies, learning environment, discipline and curricular activities. Cronbach Alpha value for each factor remained above 0.7. For each factor strongly agree and agree frequency of responses were merged into agree set of responses whereas strongly disagree and disagree frequency of responses were combined together to give rise disagree set of responses. Then percentage of agree and disagree sets of responses on each item and the factor on the whole was calculated.

The significance of difference between agree and disagree responses was worked out through paired sample t test. Independent sample t test was used to compare the responses of male and female students' opinions on each factor. Independent sample t test and One Way ANOVA was employed to report the impact of the demographic variables i.e. parents' qualification, parental profession, family income, residential status (rural, urban) on students' perception on each factor and on the whole. The results are reported in the following tables (see table 1-10).

\section{Results}

It appears from table 1 that to more than $60 \%$ respondents the building of the institution is neat and clean, cleanliness is at the priority of school and there is an ample use of IT resources in the school for academic purposes. 58\% participants revealed that Classrooms are airy, library is fully equipped with academic resources, fully functional computer lab are available with internet facility and students have no problem to use these labs for the preparation of assignments. Agree and disagree set of responses (agree mean 331.85, disagree mean 213.14, $\mathrm{t}=8.486$, $\mathrm{df}=6$, sig. 0.00o) were significantly different in the favor of agree side.

Table 2 reveals that parents supports students for completing home-based assignments, both teachers and students enjoy good mutual relationship at school as perceived by more than $57 \%$ respondents. However, there seems lack of trust among school personnel as reflected by disputes among teaching staff according to more than 53\% students. Agree and disagree groups of views were considerably tilted towards agree side (agree mean 311.00, disagree mean 232.00, $t=5.66, \mathrm{df}=3$, sig. 0.001). 
Table 1: Students' perception on infrastructure of their School

\begin{tabular}{|c|c|c|c|c|c|c|c|c|}
\hline \multirow[b]{2}{*}{ 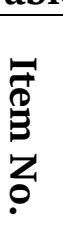 } & \multirow[b]{2}{*}{ Items } & \multirow[b]{2}{*}{$\begin{array}{l}\text { A } \\
f\end{array}$} & \multirow[b]{2}{*}{$\begin{array}{l}\text { A } \\
\%\end{array}$} & \multirow[b]{2}{*}{$\begin{array}{c}\mathrm{DA} \\
f\end{array}$} & \multirow[b]{2}{*}{$\begin{array}{l}\text { DA } \\
\%\end{array}$} & \multirow[b]{2}{*}{$\begin{array}{l}\text { Factor } \\
\text { loading }\end{array}$} & \multirow[b]{2}{*}{ 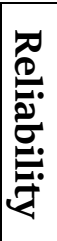 } & \multirow[b]{2}{*}{ 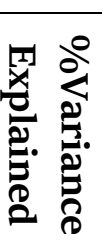 } \\
\hline & & & & & & & & \\
\hline 57 & Neat and Clean School building & 345 & $63 \%$ & 200 & $37 \%$ & 0.434 & \multirow{9}{*}{$\begin{array}{l}\circ \\
\dot{\infty} \\
\stackrel{N}{N}\end{array}$} & \multirow{9}{*}{$\begin{array}{l}\overrightarrow{0} \\
\dot{\infty} \\
\text { o }\end{array}$} \\
\hline 58 & Airy Classrooms & 318 & $58 \%$ & 227 & $42 \%$ & 0.422 & & \\
\hline 59 & Cleanliness is the priority of school & 355 & $62 \%$ & 190 & $38 \%$ & 0.350 & & \\
\hline 60 & School library is fully equipped & 316 & $58 \%$ & 229 & $42 \%$ & 0.343 & & \\
\hline 61 & $\begin{array}{l}\text { IT resources are sufficiently used in the } \\
\text { school }\end{array}$ & 354 & $65 \%$ & 191 & $35 \%$ & 0.340 & & \\
\hline 62 & Internet is available in Computer labs & 317 & $58 \%$ & 228 & $42 \%$ & 0.339 & & \\
\hline 63 & $\begin{array}{l}\text { Students are encouraged to use } \\
\text { Computer labs }\end{array}$ & 318 & $58 \%$ & 227 & $42 \%$ & 0.324 & & \\
\hline & Average & 332 & $60 \%$ & 213 & $40 \%$ & 0.364 & & \\
\hline & $\begin{array}{l}\text { Over all perception } \\
\text { Paired sample } t \text {-test }\end{array}$ & \multicolumn{2}{|c|}{$\begin{array}{l}\text { Agree Mean } \\
331.85 \\
\text { Agree } \\
\text { Standard } \\
\text { deviation } \\
18.506\end{array}$} & \multicolumn{3}{|c|}{$\begin{array}{l}\text { Disagree Mean } 213.14 \\
\text { Disagree Standard deviation } \\
18.506 \\
t=8.486 \\
\mathrm{df}=6 \\
\text { sig. .ooo }\end{array}$} & & \\
\hline
\end{tabular}

Table 2: Students' perception on nature of relationships at their school

\begin{tabular}{|c|c|c|c|c|c|c|c|c|}
\hline 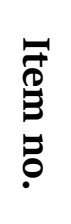 & Items & $\begin{array}{l}\text { A } \\
f\end{array}$ & $\begin{array}{c}\text { A } \\
\%\end{array}$ & $\begin{array}{c}\mathrm{DA} \\
f\end{array}$ & $\begin{array}{l}\text { DA } \\
\%\end{array}$ & $\begin{array}{l}\text { Factor } \\
\text { loading }\end{array}$ & : & 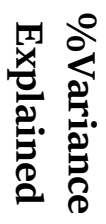 \\
\hline 53 & Coordination between parents and Teachers & 312 & $57 \%$ & 233 & $43 \%$ & .402 & \multirow{6}{*}{$\stackrel{\substack{\infty \\
w}}{\infty}$} & \multirow{6}{*}{$\dot{\varphi}$} \\
\hline 54 & Lack of trust at personnel's level & 295 & $54 \%$ & 250 & $46 \%$ & .389 & & \\
\hline 55 & $\begin{array}{l}\text { Good mutual relationship between teachers } \\
\text { and students }\end{array}$ & 315 & $58 \%$ & 230 & $42 \%$ & .385 & & \\
\hline 56 & Disputes among teaching staff & 330 & $61 \%$ & 215 & $39 \%$ & .361 & & \\
\hline & $\begin{array}{c}\text { Average } \\
\end{array}$ & 313 & $58 \%$ & 232 & $43 \%$ & .384 & & \\
\hline & $\begin{array}{l}\text { Over all perception } \\
\text { Paired sample } t \text {-test }\end{array}$ & \multicolumn{2}{|c|}{$\begin{array}{l}\text { Agree Mean } \\
311.00 \\
\text { Disagree } \\
\text { Standard } \\
\text { deviation } \\
14.35\end{array}$} & \multicolumn{3}{|c|}{$\begin{array}{l}\text { Disagree Mean } 232.00 \\
\text { Disagree Standard } \\
\text { deviation } 14.35 \\
t=5.66 \\
\mathrm{df}=3 \\
\text { sig. .001 }\end{array}$} & & \\
\hline
\end{tabular}

According to table 3 in more than $51 \%$ cases teachers are aware with the course objectives, they teach the students through different teaching strategies, frequently depend on lecture method with limited interaction ( $54 \%$ students agreed) with students their major focus is to improve the knowledge of students particularly those having difficulty in learning, the classroom teaching is marginally relevant (51\% students agreed) with the students' needs, the major focus of the teaching is to get 
through the examinations. Perception of $63 \%$ students reflects that the test scores are true reflection of the academic achievement of students. There was significance difference between agree and disagree set of responses favoring agree side (agree mean 321.88, disagree mean 223.11, $t=4.784, \mathrm{df}=8$, sig. 0.001).

Table 3: Students' perception on the effectiveness of teaching-learning process

\begin{tabular}{|c|c|c|c|c|c|c|c|c|}
\hline $\begin{array}{l}\text { 㺃 } \\
\stackrel{3}{3} \\
0 \\
0\end{array}$ & Items & $\begin{array}{l}\text { A } \\
f\end{array}$ & $\begin{array}{l}\text { A } \\
\%\end{array}$ & $\begin{array}{c}\text { DA } \\
f\end{array}$ & $\begin{array}{l}\text { DA } \\
\%\end{array}$ & $\begin{array}{l}\text { Factor } \\
\text { loading }\end{array}$ & 苞 & 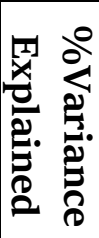 \\
\hline 1 & Using variety of teaching methods & 322 & $59 \%$ & 223 & $41 \%$ & .490 & \multirow{11}{*}{$\begin{array}{l}0 \\
\infty \\
\infty\end{array}$} & \multirow{11}{*}{$\begin{array}{l}\text { ô } \\
\text { ஸु }\end{array}$} \\
\hline 2 & Effectiveness of lecture method & 299 & $55 \%$ & 246 & $45 \%$ & .472 & & \\
\hline 3 & Awareness with course objectives & 290 & $53 \%$ & 255 & $47 \%$ & .436 & & \\
\hline 4 & $\begin{array}{l}\text { The major focus of teaching is to get through } \\
\text { the examination }\end{array}$ & 301 & $55 \%$ & 244 & $45 \%$ & .402 & & \\
\hline 5 & $\begin{array}{l}\text { Test scores truly reflect students' } \\
\text { achievement }\end{array}$ & 342 & $63 \%$ & 203 & $37 \%$ & .397 & & \\
\hline 6 & More focus in class on weak students & 370 & $68 \%$ & 175 & $32 \%$ & .386 & & \\
\hline 7 & Teaching students as a whole group & 344 & $63 \%$ & 201 & $37 \%$ & .355 & & \\
\hline 8 & $\begin{array}{l}\text { Relevance of classroom teaching with } \\
\text { students' needs }\end{array}$ & 280 & $51 \%$ & 265 & $49 \%$ & .328 & & \\
\hline 9 & $\begin{array}{l}\text { Interaction between teachers and students } \\
\text { during lecturing }\end{array}$ & 349 & $64 \%$ & 196 & $36 \%$ & $\cdot 319$ & & \\
\hline & Average & 322 & $59 \%$ & 233 & $41 \%$ & .398 & & \\
\hline & $\begin{array}{l}\text { Over all perception } \\
\text { Paired sample } t \text {-test }\end{array}$ & \multicolumn{2}{|c|}{$\begin{array}{l}\text { Agree Mean } \\
321.88 \\
\text { Agree } \\
\text { Standard } \\
\text { deviation } \\
30.97\end{array}$} & \multicolumn{3}{|c|}{$\begin{array}{l}\text { Disagree Mean } 223.11 \\
\text { Disagree Standard } \\
\text { deviation } 30.97 \\
t=4.784 \\
\mathrm{df}=8 \\
\text { sig. } 0.001\end{array}$} & & \\
\hline
\end{tabular}

Table 4 indicates that to more than 6o\% respondents students actively participate in teachinglearning activities. Such type of activities help to choose the institution for the education of their child. According to 55\% students teaching methodologies are effective. To 59\% respondents teachers and students cooperate with each other in academic matters. As per opinion of $51 \%$ participants learning environment of their school is interactive. $57 \%$ students enjoyed their stay at school. Peer tutoring is practiced in 37\% cases. There was no significance difference (agree mean 297.14, disagree mean $247.85, \mathrm{t}=1.390, \mathrm{df}=6$,sig. 0.214 ) between agree and disagree set of responses.

Table 5 depicted that more than 6o\% students were satisfied with the practical measures of school management for ensuring quality education. More than 50\% parents choose higher secondary schools for admission of their children and they focused on the students' achievement in PTM, school had effective learning program for their learners and efforts were made to recognize and solved the problems of students. There is significance difference between agree and disagree set of responses (agree mean 306.66, disagree mean 243.33, $t=2.597, \mathrm{df}=5$, sig. 0.048) favoring agree side. 
Table 4: Students' perception on school environment of higher secondary schools

\begin{tabular}{|c|c|c|c|c|c|c|c|c|}
\hline \multirow[b]{2}{*}{ 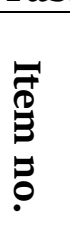 } & \multirow[b]{2}{*}{ Items } & \multirow[b]{2}{*}{$\begin{array}{l}\mathrm{A} \\
\boldsymbol{f}\end{array}$} & \multirow[b]{2}{*}{$\begin{array}{l}\mathrm{A} \\
\%\end{array}$} & & & & & \\
\hline & & & & $\begin{array}{c}\mathrm{DA} \\
f\end{array}$ & $\begin{array}{l}\text { DA } \\
\%\end{array}$ & $\begin{array}{l}\text { Factor } \\
\text { loading }\end{array}$ & : & 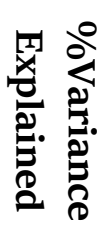 \\
\hline 12 & $\begin{array}{l}\text { Value of teaching-learning activities for } \\
\text { parents }\end{array}$ & 335 & $61 \%$ & 210 & $39 \%$ & .439 & \multirow{9}{*}{ i̊ } & \multirow{9}{*}{$\stackrel{\infty}{\text { in }}$} \\
\hline 14 & academic cooperation & 324 & $59 \%$ & 221 & $41 \%$ & .427 & & \\
\hline 16 & Effectiveness of teaching methodologies & 300 & $55 \%$ & 245 & $45 \%$ & .419 & & \\
\hline 17 & Peer tutoring practices & 200 & $37 \%$ & 345 & $63 \%$ & .389 & & \\
\hline 19 & $\begin{array}{l}\text { Active participation of students in learning } \\
\text { activities }\end{array}$ & 331 & $61 \%$ & 214 & $39 \%$ & .360 & & \\
\hline 20 & Interaction in school environment & 280 & $51 \%$ & 265 & $49 \%$ & .347 & & \\
\hline 21 & Students enjoy their stay at school & 310 & $57 \%$ & 235 & $43 \%$ & .315 & & \\
\hline & Average & 297 & $54 \%$ & 247 & $46 \%$ & .385 & & \\
\hline & $\begin{array}{l}\text { Over all perception } \\
\text { Paired sample } t \text {-test }\end{array}$ & \multicolumn{2}{|c|}{$\begin{array}{l}\text { Agree Mean } \\
297.14 \\
\text { Agree } \\
\text { Standard } \\
\text { deviation } \\
46.91\end{array}$} & \multicolumn{3}{|c|}{$\begin{array}{l}\text { Disagree Mean } 247.85 \\
\text { Disagree Standard } \\
\text { deviation } 46.91 \\
t=1.390 \\
\mathrm{df}=6 \\
\text { sig. } 0.214\end{array}$} & & \\
\hline
\end{tabular}

Table 5: Students' satisfaction with the functioning of school management

\begin{tabular}{|c|c|c|c|c|c|c|c|c|}
\hline & & & & & & & & \\
\hline 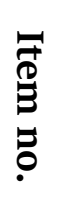 & Items & $\begin{array}{l}\text { A } \\
f\end{array}$ & $\begin{array}{l}\text { A } \\
\%\end{array}$ & $\begin{array}{c}\text { DA } \\
f\end{array}$ & $\begin{array}{l}\text { DA } \\
\%\end{array}$ & $\begin{array}{l}\text { Factor } \\
\text { loading }\end{array}$ & 串 & 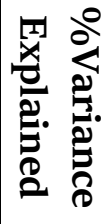 \\
\hline 22 & School management takes practical measures & 341 & $63 \%$ & 204 & $37 \%$ & .470 & & $\varphi$ \\
\hline 23 & School management focuses quality education & 327 & $60 \%$ & 218 & $40 \%$ & .421 & & 곤 \\
\hline 24 & Parents' first choice institution & 319 & $59 \%$ & 226 & $41 \%$ & .375 & & \\
\hline 25 & $\begin{array}{l}\text { PTM's goal revolves around students' } \\
\text { achievements }\end{array}$ & 295 & $54 \%$ & 250 & $46 \%$ & .331 & & \\
\hline 26 & School has effective learning program & 280 & $51 \%$ & 265 & $49 \%$ & .331 & & \\
\hline 27 & Effort to solve learning problems & 278 & $51 \%$ & 297 & $49 \%$ & .310 & 0 & \\
\hline & Average & 307 & $56 \%$ & 243 & $44 \%$ & .373 & $\stackrel{\infty}{\omega}$ & \\
\hline & $\begin{array}{l}\text { Over all perception } \\
\text { Paired sample } t \text {-test }\end{array}$ & $\begin{array}{l}\text { Agre } \\
\text { Mea1 } \\
306 . \\
\text { Agre } \\
\text { Stan } \\
\text { devi } \\
26.1\end{array}$ & $\begin{array}{l}\text { e } \\
56 \\
\text { dard } \\
\text { tion }\end{array}$ & $\begin{array}{l}\text { Disa } \\
243 \cdot \\
\text { Disa } \\
\text { devia } \\
t=2 . \\
\mathrm{df}=5 \\
\text { sig. }\end{array}$ & $\begin{array}{l}\text { ree Me } \\
3 \\
\text { ree Sta } \\
\text { tion } 34 \\
97 \\
.048\end{array}$ & $\begin{array}{l}\text { an } \\
\text { ndard } \\
30\end{array}$ & & \\
\hline
\end{tabular}


Table 6: Students' perception about discipline

\begin{tabular}{|c|c|c|c|c|c|c|c|c|}
\hline 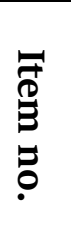 & Items & $\begin{array}{l}\text { A } \\
f\end{array}$ & $\begin{array}{l}\text { A } \\
\%\end{array}$ & $\begin{array}{c}\text { DA } \\
f\end{array}$ & $\begin{array}{l}\text { DA } \\
\%\end{array}$ & $\begin{array}{l}\text { Factor } \\
\text { loading }\end{array}$ & 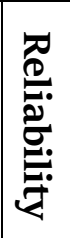 & 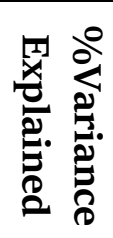 \\
\hline 31 & $\begin{array}{l}\text { Practice of rules and regulations by } \\
\text { students }\end{array}$ & 360 & $66 \%$ & 185 & $34 \%$ & .428 & \multirow{9}{*}{$\stackrel{\circ}{\text { Ǹ }}$} & \multirow{9}{*}{$\begin{array}{l}\infty \\
\dot{\infty} \\
\omega\end{array}$} \\
\hline 32 & A disciplined institution & 343 & $63 \%$ & 202 & $37 \%$ & .392 & & \\
\hline 33 & Following time table by personnel & 290 & $53 \%$ & 255 & $47 \%$ & .350 & & \\
\hline 34 & Tutorial sessions are arranged & 287 & $53 \%$ & 258 & $47 \%$ & .342 & & \\
\hline 35 & An organized institution & 297 & $54 \%$ & 248 & $46 \%$ & .337 & & \\
\hline 36 & Time carefree staff and students & 265 & $49 \%$ & 280 & $51 \%$ & .331 & & \\
\hline 37 & Students respect each other & 311 & $57 \%$ & 234 & $43 \%$ & .324 & & \\
\hline & Average & 307 & $56 \%$ & 237 & $44 \%$ & .357 & & \\
\hline & $\begin{array}{l}\text { Over all perception } \\
\text { Paired sample } t \text {-test }\end{array}$ & \multicolumn{2}{|c|}{$\begin{array}{l}\text { Agree Mean } \\
307 \cdot 57 \\
\text { Agree } \\
\text { Standard } \\
\text { deviation } \\
33.34\end{array}$} & \multicolumn{3}{|c|}{$\begin{array}{l}\text { Disagree Mean } 237.42 \\
\text { Disagree Standard } \\
\text { deviation } 33.34 \\
t=2.783 \\
\mathrm{df}=6 \\
\text { sig. } 0.032\end{array}$} & & \\
\hline
\end{tabular}

Table 7: Students' perception about co-curricular activities

\begin{tabular}{|c|c|c|c|c|c|c|c|c|}
\hline 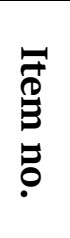 & Items & $\begin{array}{l}\text { A } \\
f\end{array}$ & $\begin{array}{l}\text { A } \\
\%\end{array}$ & $\begin{array}{c}\mathrm{DA} \\
f\end{array}$ & $\begin{array}{l}\text { DA } \\
\%\end{array}$ & $\begin{array}{l}\text { Factor } \\
\text { loading }\end{array}$ & 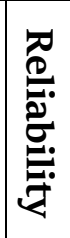 & 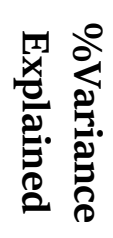 \\
\hline 64 & $\begin{array}{l}\text { Students satisfaction with co-curricular } \\
\text { activities }\end{array}$ & 355 & $65 \%$ & 190 & $35 \%$ & .406 & \multirow{7}{*}{$\dot{\dot{\nu}}$} & \multirow{7}{*}{$\begin{array}{l}\infty \\
\dot{0} \\
\dot{\infty}\end{array}$} \\
\hline 65 & $\begin{array}{l}\text { Variety of co-curricular activities are } \\
\text { observed }\end{array}$ & 327 & $60 \%$ & 218 & $40 \%$ & $\cdot 393$ & & \\
\hline 66 & $\begin{array}{l}\text { Organizing different co-curricular } \\
\text { activities on regular basis }\end{array}$ & 319 & $59 \%$ & 226 & $41 \%$ & .353 & & \\
\hline 67 & $\begin{array}{l}\text { Suggestions are always welcomed at } \\
\text { school level }\end{array}$ & 275 & $50 \%$ & 270 & $50 \%$ & $\cdot 344$ & & \\
\hline 68 & Students receive proper feedback & 353 & $65 \%$ & 190 & $35 \%$ & .307 & & \\
\hline & Average & 326 & $60 \%$ & 219 & $40 \%$ & .360 & & \\
\hline & $\begin{array}{l}\text { Over all perception } \\
\text { Paired sample } t \text {-test }\end{array}$ & \multicolumn{2}{|c|}{$\begin{array}{l}\text { Agree Mean } \\
325.80 \\
\text { Agree } \\
\text { Standard } \\
\text { deviation } \\
32.48\end{array}$} & \multicolumn{3}{|c|}{$\begin{array}{l}\text { Disagree Mean } 218.80 \\
\text { Disagree Standard } \\
\text { deviation } 32.91 \\
t=3.659 \\
\mathrm{df}=4 \\
\text { sig } 0.022\end{array}$} & & \\
\hline
\end{tabular}

According to table 6, in more than 60\% cases rules and regulations are practiced in their school and it was not difficult for a person to follow the discipline. According to more than $50 \%$ respondents time table was followed by the personnel, tutorial session were regularly arranged, school was an 
organized institution and students respect each other. 51\% students disagreed with the statement that there were time carefree staff and students in this institutions. There was a significance difference between agree and disagree set of response favoring agree side (agree mean 307.57, disagree mean 237.42, $t=2.783, \mathrm{df}=6$, sig. 0.032 ).

As table 7 indicated that students were satisfied with the co-curricular activities, variety of cocurricular activities were practiced and students received proper response from school management (6o\% respondents). 59\% respondents were satisfied with the organizing and provisions for cocurricular activities on regular basis. There was significance difference between agree and disagree set of responses favoring agree side (agree mean 325.8o, disagree mean 218.8o, $\mathrm{t}=3.659$, $\mathrm{df}=4$, sig. 0.022 ).

Table 8: Impact of gender on students' perception regarding effectiveness of HSS

\begin{tabular}{|c|c|c|c|c|c|c|c|c|}
\hline $\begin{array}{c}\text { Sr. } \\
\text { No }\end{array}$ & Gender & $\mathbf{N}$ & Mean & $\begin{array}{c}\text { Standard } \\
\text { Deviation }\end{array}$ & $\begin{array}{c}\text { Standard } \\
\text { Error Mean }\end{array}$ & $\mathbf{t}$ & df & Sig. (2-tailed) \\
\hline \multirow{2}{*}{1} & Male & 300 & 1.54 & .499 & .020 & 3.038 & 1098 & 0.002 \\
\cline { 2 - 9 } & Female & 245 & 1.45 & .499 & 0.002 & \\
\hline
\end{tabular}

Regarding table 8 there is significant difference between the perception of male and female higher secondary school students regarding effectiveness of higher secondary schools.

Table 9: Impact of locality on students' perception regarding effectiveness of HSS

\begin{tabular}{|c|c|c|c|c|c|c|c|c|}
\hline $\begin{array}{c}\text { Sr. } \\
\text { No }\end{array}$ & Gender & $\mathbf{N}$ & Mean & $\begin{array}{c}\text { Standard } \\
\text { Deviation }\end{array}$ & $\begin{array}{c}\text { Standard } \\
\text { Error Mean }\end{array}$ & $\mathbf{t}$ & df & Sig. (2-tailed) \\
\hline \multirow{2}{*}{1} & Rural & 244 & 1.48 & .500 & .023 & 0.917 & 1096 & 0.001 \\
\cline { 2 - 9 } & Urban & 301 & 1.51 & .500 & \\
\hline
\end{tabular}

Regarding table 9 there is significant Impact of locality on students' perception regarding characteristics of their institution. Difference is significant 0.001 between both group of students from rural areas and urban areas.

Table 10: Impact of class on students' perception about higher secondary school

\begin{tabular}{|c|c|l|c|c|c|c|c|c|c|}
\hline $\begin{array}{c}\text { Sr. } \\
\text { No }\end{array}$ & $\begin{array}{c}\text { Item } \\
\text { No. }\end{array}$ & \multicolumn{1}{|c|}{ Statement } & $\begin{array}{c}\text { Class } \\
\text { (I) }\end{array}$ & $\begin{array}{c}\text { Class } \\
\text { (J) }\end{array}$ & $\begin{array}{c}\text { Mean } \\
\text { Difference }\end{array}$ & $\begin{array}{c}\text { Standard } \\
\text { Error }\end{array}$ & DF & F & Sig. \\
\hline 1 & 27 & $\begin{array}{l}\text { Learning problems of } \\
\text { students in this institution } \\
\text { are identified and worked } \\
\text { out. }\end{array}$ & F.Sc & I.com & .286 & .099 & 3 & 2.940 & 0.024 \\
\hline
\end{tabular}

Table 10 showed that the children whose class is F.Sc have more perception about institution then those whose group of class is I.com. There is significant difference (sig. $=0.024$ ) between both group.

\section{Findings of the Study}

1. More than sixty percent respondents agreed that institution building is neat and clean, priority of school is cleanliness, and IT sources are sufficiently used in the school for academic purposes. More than fifty percent respondents revealed that classrooms are airy, library is fully equipped, 
availability of fully functional computer lab with internet facility and students are allowed to use computer for assignments. There was a significance difference between agree and disagree set of responses (agree mean 331.85, disagree mean 213.14, $\mathrm{t}=8.486, \mathrm{df}=6$, sig. 0.000).

2. More than fifty four percent students agreed that coordination between parents and teachers for home tasks for students, lack of trust among personnel, good student teachers relationship, and teaching staff dispute. There was significance difference between agree and disagree set of response (agree mean 311.00, disagree mean 232.00, $t=5.66, \mathrm{df}=3$, sig. 0.001 ).

3. More than fifty percent students agreed that practice of different teaching methods, improvement of knowledge, awareness of course objectives, assignment tasks, teachers realize when students did not understand. Whereas more than sixty percent students agreed that test questions enhance classrooms teaching, weak students were teachers' priority, teachers explains lectures, interactive lectures by teachers. There was significance difference between agree and disagree set of response (agree mean 321.88, disagree mean 223.11, $t=4.784, \mathrm{df}=8$, sig. 0.001 ).

4. More than sixty-three percent disagreed with the statement that peer tutoring was common. More than sixty percent agreed that parents focused on school activities and students participate in learning process. More than fifty percent agreed that teachers helped each other, teacher adopt lecture method, learning environment of class was interactive and students enjoyed at school. There was no significance difference between agree and disagree set of responses (agree mean 297.14, disagree mean 247.85, $\mathrm{t}=1.390$, $\mathrm{df}=6$, sig. 0.214 ).

5. More than sixty percent students agreed that leadership believe in practical steps and quality. More than fifty percent agreed that parents' first choice institution, students achievement in PTM's goal, school has effective learning program and problems of students are recognized and worked out. There is significance difference between agree and disagree set of responses (agree mean 306.66, disagree mean 243.33, $\mathrm{t}=\mathbf{2 . 5 9 7} \mathrm{df}=5$, sig. 0.048).

6. More than sixty percent respondents practice of schools rules and regulations and discipline is not difficult to follow. More than fifty percent respondents follow the time table, tutorial session were regularly arranged, an organized institutions and students respect each other. Fifty one percent students disagreed with the statement that time carefree staff and students in these institutions. There was a significance difference between agree and disagree set of response (agree mean 307.57, disagree mean 237.42, $\mathrm{t}=2.783$, $\mathrm{df}=6$, sig. 0.032).

7. More than sixty percent respondents were satisfied with the curricular activities, variety of cocurricular activities were practiced, and students received proper response. Fifty-nine percent respondents agreed that there were sufficient provisions for co-curricular activities. There was significance difference between agree and disagree set of responses (agree mean 325.80, disagree mean $218.80, t=3.659, \mathrm{df}=4$, sig. 0.022 ).

8. Results of independent t-test statistics regarding the comparison of male and female secondary school students' opinions regarding the school effectiveness were significant at $(\mathrm{p}<0.05)$ which shows that there was statistically significant difference of opinions of students about effectiveness.

9. There was significant difference (o.002) between the perception of male and female students of higher secondary school regarding effectiveness of higher secondary schools.

10. There was significant difference (0.001) between group of students from rural areas and urban areas.

11. The result of ANOVA described that children whose class is FSc have more perception about institution then those whose group of class is I.com. There is significant difference (sig $=0.024)$ between both group. 


\section{Conclusions and Discussion}

The results of the survey indicate that more than 6o percent of male students agreed that the learning process of teaching is an important factor in the effectiveness of the school and played an important role in maintaining the quality of education at secondary school level. While more than fifty four percent of the students thought that creating an effective learning environment for effective educational outcomes in institutions. Meanwhile, most students were in favor of the institution's assessment process and infrastructure playing a vital role in the institution's progress. From the results of study, it can be inferred that male students need an effective learning environment, well-planned teaching strategies and co-curricular activities in their institution to achieve good academic results. Scheerens (2000) research also confirmed the results that when students perceive their teachers as supportive; reduces disruptive behavior and increases students' perceptions of successful interactions with their teachers. A positive school environment, i.e. a classroom where students feel supported and where order is maintained, generally lends itself to higher student academic performance. Teachers who support their students academically and emotionally, who are kind to them, and who help build collaboration between them, tend to elicit better academic functioning from their students (Dochy, Segers, \&Sluijsmans 1999). Findings of the study reveal the comparison of male and female students' opinions regarding the secondary school students. The findings show statistically significant results between student and female student opinions.

1. The study revealed that there were lot of disputes among staff and personnel. It is recommended that there should be better relationship among staff and personnel for better work environment.

2. The study confirmed the results that there was no peer tutoring in institutions. It is suggested that there should be peer tutoring for the academic development of students.

3. It is discovered that mostly teachers teach without activities in the classrooms. It is suggested that there should be activities based teaching at higher secondary level.

4. It is recommended that there should be counseling and guidance for academic and intellectual development of students.

5. It is suggested that there should be improvement in learning environment of higher secondary schools.

\section{References}

Ali, H., Ullah, S., Najam ul Kashif \& Husssain, B. (2016). Analysis of Grade Inflation at Secondary School Level: Case Study of Board of Intermediate and Secondary Education, Multan,. Pakistan Journal of Social Sciences (PJSS), 36(2), 1011-1022.

Barton, P. E. (2005).One-third of a nation: Rising dropout rates and declining opportunities. Educational Testing Service, Policy Information Center, Princeton; NJ.

Brophy, J. (2004). Motivating Students to Learn, Routledge, New York. https://doi.org/ $10.4324 / 9780203858318$

Blair, M. (2002). Effective school leadership: The multi-ethnic context. British Journal of Sociology of Education, 23(2), 179-191.

Dochy, F. J. R. C., Segers, M., \&Sluijsmans, D. (1999). The use of self-, peer and co-assessment in higher education: A review. Studies in Higher education, 24(3), 331-350.

Fredericks, J. A., Blumenfeld, P. C., \& Paris, A. H. (2004). School engagement: Potential of the concept, state of the evidence. Review of Educational Research, 74, 59 - 109.

Govt. of Pakistan (March 2018). Pakistan Education Statistics 2016-17. Ministry of Federal Education and Professional Training.

Johnson, R. B., \& Christensen, L. (2012). Educational research: Quantitative, qualitative, and mixed approaches. Sage publications. 
Rutter, M., Maughan, B., Mortimore, P., Ouston, J., \& Smith, A. (1979). Fifteen thousand hours: Secondary schools and their effects on children. London, England: Open Books.

Rutter, M., Maughan, B., Mortimore, P., Ouston, J., \& Smith, A. (1979). Fifteen thousand hours: Secondary schools and their effects on children. London, England: Open Books.

Roland, E., \& Galloway, D. (2004). Professional cultures in schools with high and low rates of bullying. School Effectiveness and School Improvement, 15(3-4), 241-26o.

Saleem, F. (2010).Development of School Effectiveness Model. Journal of Research \& Reflections in Education (JRRE), 4(2).

Scheerens, J. (2000), Improving school effectiveness: Fundamentals of educational planning,

Scheerens, J. (2016). Educational effectiveness and ineffectiveness.A critical review of the knowledge base. Dordrecht, The Netherlands: Springer.

Teddlie, C., \& Reynolds, D. (2000). The international handbook of school effectiveness research. London: FalmerPress.United Nations Educational Scientific and Cultural Organization, De Fonteyn, Paris. Retrieved from http:www.unesco.org//iiep

Wallin, J. (2003). Improving School Effectiveness. ABAC Journal, 23(1): 61-72.

Yusuf, L. A. and Alabi, C. O. (2013).Enhancing School Effectiveness in the Universal Basic Education (UBE) Programmein Nigeria: Issues and Implications.Journal of Education, Arts and Humanities, 1(3):022-026. 